\title{
DNA knot malleability in single-digit
}

\section{nanopores}

Rajesh Kumar Sharma, ${ }^{\dagger, \uparrow, \uparrow}$ Ishita Agrawal, ${ }^{\S}$ Liang Dai, $\|$ Patrick Doyle, ${ }^{*,+, \perp}$ and Slaven Garaj*,†, $\boldsymbol{\Phi}, \S$

$\dagger$ Department of Physics, National University of Singapore, Singapore

$\ddagger$ Singapore-MIT Alliance for Research and Technology Centre, Singapore

ฯCentre for Advanced 2D Materials, National University of Singapore, Singapore

$\S$ Department of Biomedical Engineering, National University of Singapore, Singapore

||Department of Physics, City University of Hong Kong, Kowloon, Hong Kong, P.R. China $\perp$ Department of Chemical Engineering, Massachusetts Institute of Technology, Cambridge, MA, United States

E-mail: pdoyle@mit.edu; slaven@nus.edu.sg

\section{Nanopore Fabrication}

We purchased wafers (LPCVD grown $300 \mathrm{~nm}$ thick low stress silicon nitride on $525 \mu \mathrm{m}$ thick silicon wafers) from Cornell NanoScale Science and Technology Facility, Ithaca, NY, USA. Windows of free-standing silicon nitride membranes were fabricated using standard photolithography and deep reactive ion etching of a wafer, followed by the anisotropic wet etching of silicon in $33 \% \mathrm{KOH}$ solution.

Wafers were further patterned using electron beam lithography to fabricate one minimembrane of diameter $500 \mathrm{~nm}$ in each freestanding silicon nitride membrane. Deep reactive ion etching was used to thin the membrane further down to a thickness of $20 \mathrm{~nm}$. The whole 
wafer was diced to segregate individual chips. Each chip was drilled using a focused electron beam in a JEOL $2010 \mathrm{~F}$ electron microscope operating at $200 \mathrm{kV}$.

\section{Nanopore cleaning and assembly}

Nanopores were treated with acid piranha $\left(7: 3:: \mathrm{H}_{2} \mathrm{SO}_{4}: \mathrm{H}_{2} \mathrm{O}_{2}\right)$ for 30 minutes and then rinsed with ultrapure water 4-5 times, followed by blow drying with nitrogen gas. Each membrane with a nanopore was placed inbetween two fluidic compartments filled with $1 \mathrm{M}$ potassium chloride ( $\mathrm{KCl}$ ) buffered with $10 \mathrm{mM}$ Tris and $1 \mathrm{mM}$ ethylenediaminetetraaceticacid (EDTA), $\mathrm{pH}=8.2$, in such a way that the nanopore remained the only connection between the two compartments. Two freshly regenerated $\mathrm{Ag} / \mathrm{AgCl}$ electrodes were connected to the compartments for ionic conductivity measurements through the nanopore.

\section{Nanopore Characterisation}

To confirm the sizes of the nanopores measured using transmission electron microscopy (TEM), we characterise the nanopores based on their ionic conductance. We measure the

open pore conductance of our nanopores and compare them with the expectations from the Ohmic formula (Figure 1). The observed values for follow well the expectation from the serial contribution of the channel resistance and the access resistance, and fit well the geometrical parameters of the nanopores:

$$
R_{\text {total }}=R_{\text {channel }}+R_{\text {access }}=\frac{D^{2} \pi}{4 L \sigma}+\frac{D}{\sigma}
$$

where $D$ is the diameter of the nanopores, $L=(15 \pm 2) \mathrm{nm}$ is the thickness of the membrane (length of the channel) and $\sigma$ is the conductivity of the solution. 


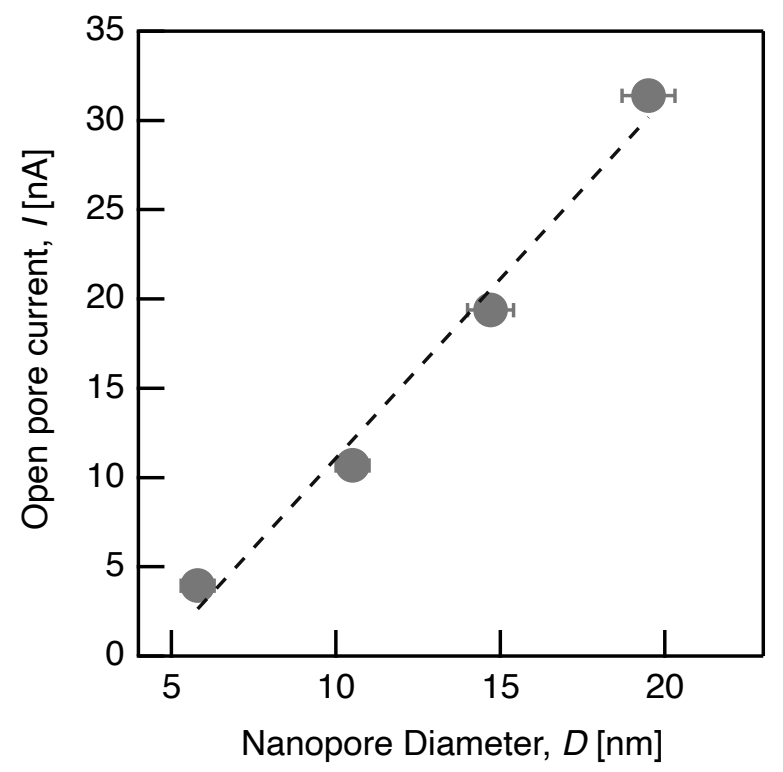

Figure 1: Open pore currents of the four nanopores used in this study with diameters $D=$ $(5.8 \pm 0.5) \mathrm{nm},(10.5 \pm 0.5) \mathrm{nm},(14.7 \pm 0.7) \mathrm{nm}$, and $(19.5 \pm 0.8) \mathrm{nm}$, fabricated in $\mathrm{SiN}_{\mathrm{X}}$ freestanding membranes of thickness $L=(15 \pm 2) \mathrm{nm}$. The observed conductivities match well the predictions the standard Ohmic conductance model with access resistance (dashed line).

\section{DNA packing in nanopores}

For each DNA translocation event, we measure the maximum number of double-stranded DNA (ds-DNA) molecules that are packed within the pore concurrently. The values are calculated by dividing the maximum drop in the current blockade signal, with the average current blockade of a single-file DNA molecule, and rounded to the nearest integer. Figure 2 shows the occurrence of the DNA translocation events with given maximal number of DNA molecules packing within the pore, presented for the nanopores with diameters $D=5 \mathrm{~nm}$ and $D=20 \mathrm{~nm}$. Knots - based on the their complexity, order, and circular or linear nature of the host DNA molecule - could have any number of concurrent DNA molecules larger that 3 .

In $D=5 \mathrm{~nm}$ nanopore, we observe no more than 6 compacted DNA molecules packing the pore at the same time, and in $D=20 \mathrm{~nm}$ pore we observe up to 14 DNA molecules. Those results are consistent with simple geometrical expectations for close packing of ds- 


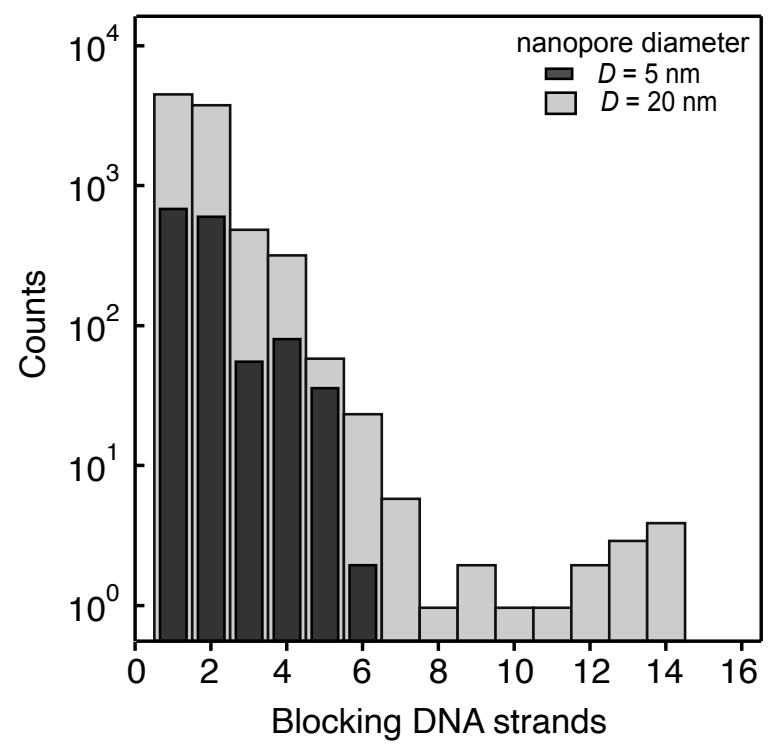

Figure 2: Histogram showing the frequency of DNA translocation events with a given maximum number DNA strands occupying the nanopore at the same instant, measured for nanopores with diameters $D=5 \mathrm{~nm}$ and $D=20 \mathrm{~nm}$. Due to geometrical exclusion, at most 6 DNA strands could occupy $D=5 \mathrm{~nm}$ nanopore at the same time; and at most 14 DNA strands could occupy $D=20 \mathrm{~nm}$ nanopore.

DNA filaments, approximated as cylinders with diameter $D_{D N A}=2 \mathrm{~nm}$.

\section{Analytical Sliding Model}

Here we describe the sliding models used for analysing the effect of sliding of DNA knots during translocation through nanopores. If there is a non-negligible sliding of knots, it will lead to different bias parameters (sliding parameter) for circular and linear DNA, since some of the knots will slip out of the linear molecules, while the knots in circular DNA cannot escape. Also, the sliding will affect the proportion of knotted molecules for linear DNA $P_{\text {lin }}$, because some knots will not be detected due to nanopore-induced slip-off. Hence $P_{\text {lin }} / P_{\text {cir }}$ ratio is expected to reduce with increase sliding. Here, we develop a simple sliding model that would quantify and correlate the above effects to a microscopic parameter and to each other. We start by exploring a discrete model, and then generalise it to continuous model. 


\section{Discrete model}

In the model, we start with the following assumptions:

1. we divide DNA molecule into $N$ segments with length $\delta$, such that the total length of the molecule is $L_{D N A}=N \cdot \delta$.

2. We assume that each segment $\delta$ has average knot density of $1 / N$. This corresponds to one knot per DNA molecule, which is sensitive enough assumption.

3. For each $n$-th segment entering the pore, we define probability $p$ that a knot will slide to the next segment with index $(n+1)$.

4. Even if the knot slides to position $n+1$, it will again have a chance $p$ to continue sliding to the next segment.

5. probability not sliding off from a segment is $q=1-p$

After passing through the pore, the density of knots in each segment will be:

$$
\begin{gathered}
c_{1}=\frac{q}{N}=\frac{1-p}{N} \\
c_{2}=\left(\frac{p}{q} c_{0}+1\right) \cdot \frac{q}{N}=\frac{1-p^{2}}{N} \\
c_{3}=\left(\frac{p}{q} c_{1}+1\right) \cdot \frac{q}{N}=\frac{p c_{1}+q}{N}=\frac{1-p+p-p^{3}}{N}=\frac{1-p^{3}}{N} \\
\cdots \\
c_{n}=\frac{1-p^{n}}{N}
\end{gathered}
$$

\section{Continuous Model}

Now we extend the model to continuous case. We assume that the sliding of a knot by distance $\Delta x$ is given by a probability density distribution $\rho(\Delta x)$. Such a distribution should 
have the following properties:

$$
\rho(x+\delta)=\rho(x) \cdot \rho(\delta)
$$

If we assume $\delta$ is a very small interval, we could employ Taylor expansion:

$$
\rho(x+\delta) \approx \rho(x)+\frac{\mathrm{d} \rho}{\mathrm{d} x} \delta
$$

Combining the last two equations, we get:

$$
\begin{gathered}
\frac{\mathrm{d} \rho}{\mathrm{d} x}=\rho(x)(\rho(\delta)-1) \\
\frac{\mathrm{d} \rho}{\rho}=\mathrm{d} x \frac{\rho(\delta)-1)}{\delta}
\end{gathered}
$$

Integrating both sides:

$$
\rho=\rho_{0} \exp \left(-x / \lambda_{S}\right)
$$

where $\rho_{0}$ is the normalisation coefficient, and $\lambda_{s} \equiv \frac{\delta}{1-\rho(\delta)}$.

Normalising the distribution:

$$
\int_{0}^{\infty} \rho(x) \mathrm{d} x=1 \Longrightarrow \rho_{0}=\frac{1}{\lambda_{S}}
$$

we get the final equation:

$$
\rho=\frac{1}{\lambda_{S}} \exp \left(-\frac{x}{\lambda_{S}}\right)
$$

We refer to $\lambda_{S}$ as the "mean sliding distance", a measure of efficiency of the sliding process. It is phenomenological parameter that encompasses microscopic mechanism responsible for sliding, which we do not wish to explore at this point.

Now we want to calculate the knot density distribution. We will employ limit of the discrete model when the size of the segment $\delta \rightarrow 0$, number of segments $N \rightarrow \infty$ and $x=N \cdot \delta$. 
First, we calculate the probability of knots slipping out of the segment $\delta$ :

$$
p_{\delta}=1-\int_{0}^{\delta} \rho(x) \mathrm{d} x=\cdots=\exp \left(-\frac{\delta}{\lambda_{S}}\right)
$$

Combining equations 2 and 4 we get (with discrete limit assumptions) and assuming initial (pre-translocation) knot density $c_{0}$ :

$$
\begin{gathered}
c_{N}=c_{0}\left(1-\exp \left(-\frac{\delta N}{\lambda_{S}}\right)\right) \\
c(x)=\frac{1}{L_{D N A}}\left(1-\exp \left(-\frac{x}{\lambda_{S}}\right)\right)
\end{gathered}
$$

Here we assumed that initially (before nanopore translocation) we have one knot per DNA molecule, i.e. $c_{0}=1 / L_{D N A}$, where $L_{D N A}$ is the length of the DNA molecule.

Now we could calculate and compare the relevant parameter that are used in our experiments.

\section{Linear DNA}

Total number of remaining knots in the linear DNA molecules after translocation (where some initial knots slid-off):

$$
\begin{gathered}
N_{k n o t}^{(l i n)}=\int_{0}^{L} c(x) \mathrm{d} x \\
N_{k n o t}^{(l i n)}=1-\Lambda+\Lambda \exp (-1 / \Lambda)
\end{gathered}
$$

where we have defined lambda to be:

$$
\Lambda=\frac{\lambda_{S}}{L}
$$


Number of knots in the first $80 \%$ of the molecule:

$$
N_{0-80}^{(\operatorname{lin})}=\int_{0}^{0.8 L} \mathrm{~d} x c(x)=0.8+\Lambda \exp \left(-\frac{0.8}{\Lambda}\right)-\Lambda
$$

Number of molecules in the last $20 \%$ of the linear molecule:

$$
N_{80-100}^{(\text {lin })}=\int_{0.8 L}^{L} \mathrm{~d} x c(x)=0.2+\Lambda\left(\exp \left(-\frac{1}{\Lambda}\right)-\exp \left(-\frac{0.8}{\Lambda}\right)\right)
$$

Sliding parameter:

$$
B_{\text {lin }} \equiv 5 \frac{N_{80-100}^{(\text {lin })}}{N_{k n o t}^{(l i n)}}=\frac{1+5 \Lambda\left(\exp \left(-\frac{1}{\Lambda}\right)-\exp \left(-\frac{0.8}{\Lambda}\right)\right)}{1-\Lambda+\Lambda \exp (-1 / \Lambda)}
$$

\section{Circular DNA}

In the last $20 \%$ segment of the molecule, all the slid knots accumulate. We use equation 7 :

$$
N_{80-100}^{(c i r)}=1-N_{0-80}^{(l i n)}=0.2+\Lambda-\Lambda \exp \left(-\frac{0.8}{\Lambda}\right)
$$

The total number of knots in circular DNA:

$$
N_{\text {knot }}^{(c i r)}=1
$$

since there is no loss of knots due to sliding.

The bias parameter due to sliding would then be given by:

$$
B_{c i r} \equiv 5 \cdot \frac{N_{80-100}^{(c i r)}}{N_{k n o t}^{(c i r)}}=1+5 \Lambda-5 \Lambda \exp \left(-\frac{0.8}{\Lambda}\right)
$$

Now we could calculate how the relative prevalence of knots between circular and linear DNA molecules, $r_{c l}$ changes upon DNA translocation with sliding: 


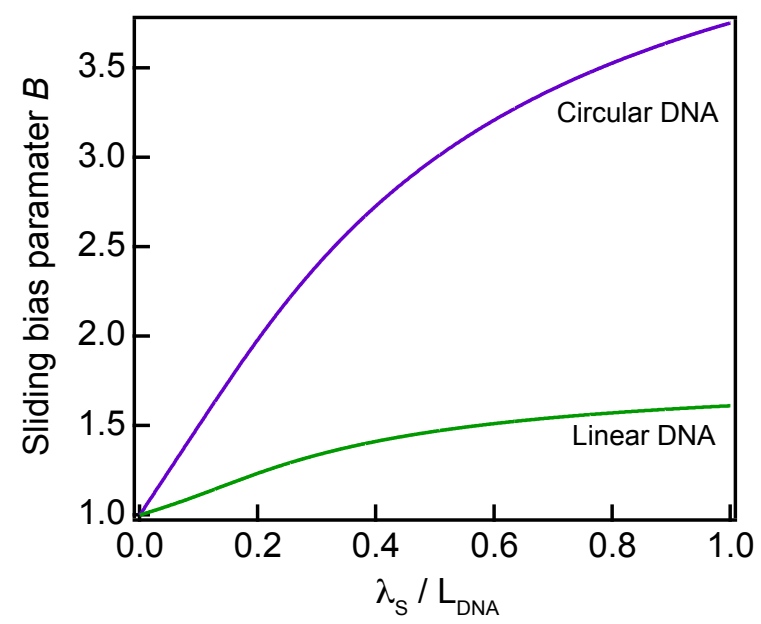

Figure 3: Bias parameters from sliding in circular and linear DNA as a function of relative sliding length $\Lambda=\lambda_{S} / L$, ratio of sliding length $\lambda_{S}$ and length of DNA $L_{D N A}$.

$$
r_{c l}=P_{c i r} / P_{l i n}=\alpha \frac{N_{k n o t}^{(c i r)}}{N_{k n o t}^{(l i n)}}=\alpha \frac{1}{1-\Lambda+\Lambda \exp (-1 / \Lambda)}
$$

where $P_{\text {cir }}$ and $P_{\text {lin }}$ are proportion of detected knotted molecules in circular and linear population, respectively; $N_{k n o t}^{(c i r)}$ and $N_{k n o t}^{(l i n)}$ are the number of detected knotted molecules in in circular and linear population, respectively; and $\alpha \propto \frac{N^{(\text {lin })}}{N^{(\text {cir })}}$ is normalisation factor that is proportional to the ratio of the total number of linear to circular DNA molecules in our sample, knotted or otherwise. We use $\alpha$ as normalisation parameter such that $r_{c l}\left(\lambda_{S}=0\right)=1$ when there is no sliding, for large pores.

The predicted difference of knot dynamics in linear and circular DNA molecules - encoded in sliding parameters $B_{\text {cir }}$ and $B_{\text {lin }}$ (see Figure 3 ), and relative knot prevalence $r_{c l}$ - allows us to deduce mean sliding length $\lambda_{S}$ and test the consistency of the sliding hypothesis. 